being available in a freeze-dried form ${ }^{7}$. Since 1974 a large number of antigens relevant to human and veterinary immunization have been incorporated in liposomes ${ }^{8}$ : the list ${ }^{6}$ includes diphtheria toxoid, Plasmodium falciparum antigens, synthetic peptides for hepatitis B and footand-mouth disease vaccines, cholera toxin and Streptococcus mutans cell-wall antigens. Studies ${ }^{6}$ in numerous laboratories have shown that optimal immunization with a given antigen can be achieved if the structural characteristics of liposomes, the way in which the antigen is accommodated (if needed together with other immunopotentiating agents) and the route and schedule of administration are judiciously chosen. Also, targeting ${ }^{6}$ of antigens to immunocompetent cells by means of liposomes coated with cell-specific ligands is likely to improve immunogenicity considerably.

It follows that the type of liposomes used by Epstein et al. ${ }^{1}$ may have contributed to the poor immune response to gp340 in the animal species used. As the liposomes were made ${ }^{2,3}$ of $\mathrm{L}$ - $\alpha$-phosphatidylcholine only, they would be expected, on reaching ${ }^{6}$ the circulating blood from the peritoneal cavity, to rapidly disintegrate through the action of high-density lipoproteins which remove phospholipid molecules from vesicles devoid of cholesterol ${ }^{6}$. Thus, only a fraction of the original dose of liposomes still containing the antigen would have reacted with immunocompetent cells. Liposomal size also controls vesicle stability as well as uptake by tissues, including lymph nodes $^{6}$. There are other variables ${ }^{6}$ which affect liposomal adjuvanticity (for example, membrane fluidity and surface charge), but of particular interest is the finding 9 that covalent attachment of antigens on the liposomal surface further augments immunogenicity and this may be applicable to gp340. It is unfortunate that the limited number of tamarins available has prevented Epstein et al. ${ }^{1}$ from evaluating the many possible versions of their liposomal vaccine.

\section{GREGORY GREGORIADIS}

Medical Research Council Group, Academic Department of Medicine, Royal Free Hospital School of Medicine, Pond Street, London NW3 2QG, UK

1. Epstein, M. A., Morgan, A. J., Finerty, S., Randle, B. J. \& Kirkwood, J. K. Nature 318, 287-289 (1985)

2. Morgan, A. J., Epstein, M. A. \& North, J. R. J. med. Virol. 13, 281-292 (1984)

3. North, J. R., Morgan, A. J., Thompson, J. L. \& Epstein, M. A. Proc. natn. Acad. Sci. U.S.A. 79, 7504-7508 (1982)

4. Epstein, M. A., Randle, B. J., Finerty, S. \& Kirkwood, J. K. Clin. exp. Immun. (in the press)

5. Beale, A. J. Nature 318, 230 (1985)

6. Gregoriadis, G. Trends Biotechnol 3, 235-241 (1985).

7. Kirby, C. \& Gregoriadis, G. Biotechnology 2(11), 979-984 (1984).

8. Allison, A. C. \& Gregoriadis, G. Nature 252, 252 (1974)

9. Snyder, S. L. \& Vannier, W. E. Biochim. biophys. Acta 772, 288-294 (1984).

\section{Life history correlations and demography}

IN a recent paper, Harvey and Zammuto ${ }^{1}$ pointed out that variation in age at first reproduction among female mammals is strongly correlated with expectation of life at birth, even after the effects of body size have been removed. We wish to make three points about the interpretation of that correlation. The second and third are of wider interest as they are relevant to a number of other studies that present relationships between life history variables.

First, expectation of life at birth is the sum of two components, pre-reproductive lifespan and reproductive lifespan. The correlation is therefore between pre-reproductive lifespan (age at first reproduction) and the sum of the two parts (expectation of life at birth). The same is true of a similar plot for iteroparous plant species given by Harper (ref. 2, page 688 ) in which age at first reproduction is plotted against lifespan. It is not surprising that the sum is correlated with one of its parts, irrespective of effects of body size. This complication can be removed by correlating age at first reproduction with reproductive lifespan rather than total lifespan. For Harvey and Zammuto's case ${ }^{1}$, the correlation is highly significant even with the effects of body size removed by partial correlation $(r=0.76, n=25, P<0.001)$.

Our second point follows because in a constant-sized population, natality must be balanced by mortality. This makes correlations between at least some components of life history inevitable, and a zero correlation between any two components a priori unlikely. In the above case, change in life expectation could be balanced by variation in litter size, interbirth interval or age at first reproduction. In fact, as Harvey and Zammuto pointed out, age at first breeding seemed to be the most relevant variable.

To make this point formally, consider a sexual population of constant size and age structure, and sexual symmetry. Let $\pi$ be the probability of surviving to reproduce, $\mu$ the mean adult mortality per year, $L$ the expected reproductive lifespan and $M$ the average number of offspring produced by a female chosen at random from those reproducing in a given year. Then

$$
\pi L M=\pi M / \mu=2
$$

This relationship could explain the results of a number of studies. Stearns ${ }^{3}$ points out a relationship between the ratio of juvenile to adult mortality $(=(1-\pi) L)$ and number of breeding seasons $(L)$, while Tinkle $^{4}$ and $\mathrm{O}^{\prime}$ Connor ${ }^{5}$ report one between fecundity $(M)$ and survivorship $(1-\mu)$. The correlation reported above might also be explained in the same way because it is between reproductive lifespan $(L)$ and age at first reproduction, which may be related inversely to the probability of survival to first breeding $(\pi)$. This case is more tenuous as it involves another relationship in addition to equation (1). Harper's ${ }^{2}$ correlation for iteroparous plant species (see above) may also be of this kind.

Unless other variables adjust to compensate, the relationships reported are in each case an inevitable demographic consequence of a net growth rate of zero. When variables are related by an exact formula such as equation (1), it is barely possible for each pair of variables to be uncorrelated. Knowing that species differ in longevity, for example, therefore makes it inevitable that some pairs of variables will be correlated.

Our final point is that it is useful to distinguish between two kinds of explanation for any pattern that holds between life history variables: adaptive and nonadaptive. The distinction was implied but not made explicit in the final paragraph of Harvey and Zammuto's paper'. They pointed out that, among Columbian ground squirrels, increase in food supplies results in earlier reproduction and higher mortality. Harvey and Zammuto considered these populations to differ in age at first breeding and reproductive lifespan for non-adaptive reasons, while interspecies differences were attributed to adaptive reasons. After showing that, irrespective of size, genera with later ages at first reproduction had lower mortality rates, they stated that "this calls for an explanation in terms of the costs and benefits of reproduction at different ages". Their reason for making this claim was that the correlation seemed to imply that some genera delay reproduction beyond the time when they become physically capable of bearing viable young. Only further work can determine whether this is so.

Life histories are often seen as strategies of animals. They may be more profitably viewed as consequences of animals' actions (which may be evolved strategies), environmental effects and demographic constraints.

We thank Andrew Watkinson and Mark Ridley for useful comments.

WilliaM J. SUTHERLAND

School of Biological Sciences

University of East Anglia,

Norwich NR4 7TJ, UK

\section{Alan GRAFEN}

PAUL H. HARVEY

Department of Zoology,

University of Oxford,

South Parks Road,

Oxford OX1 3PS, UK

1. Harvey, P. H. \& Zammuto, R. M. Nature 315, 319-320 (1985)

2. Harper, J. L. Population Biology of Plants (Academic, London, 1977).

3. Stearns, S. C. Q. Rev, Biol 51, 3-47 (1976)

4. Tinkle, D. W. Am. Nat. 103, 501-516 (1979).

5. O'Connor, R. J. in Behavioural Ecology, Ecological Consequences of Adaptive Behaviour (eds Sibley, R. M. \& Smith, R. H.) 105-142 (Blackwell, Oxford, 1985). 